\title{
Direct measurement of the $W$ boson decay width
}

V. M. Abazov, ${ }^{23}$ B. Abbott,${ }^{57}$ A. Abdesselam,${ }^{11}$ M. Abolins,${ }^{50}$ V. Abramov, ${ }^{26}$ B. S. Acharya, ${ }^{17}$ D. L. Adams,${ }^{55}$ M. Adams ${ }^{37}$ S. N. Ahmed, ${ }^{21}$ G. D. Alexeev, ${ }^{23}$ A. Alton,${ }^{49}$ G. A. Alves, ${ }^{2}$ E. W. Anderson, ${ }^{42}$ Y. Arnoud, ${ }^{9}$ C. Avila,${ }^{5}$ M. M. Baarmand, ${ }^{54}$ V. V. Babintsev ${ }^{26}$ L. Babukhadia, ${ }^{54}$ T. C. Bacon ${ }^{28}$ A. Baden, ${ }^{46}$ B. Baldin, ${ }^{36}$ P. W. Balm,${ }^{20}$ S. Banerjee,${ }^{17}$ E. Barberis ${ }^{30}$ P. Baringer, ${ }^{43}$ J. Barreto, ${ }^{2}$ J. F. Bartlett ${ }^{36}$ U. Bassler,${ }^{12}$ D. Bauer ${ }^{28}$ A. Bean ${ }^{43}$ F. Beaudette, ${ }^{11}$ M. Begel,${ }^{53}$ A. Belyaev,${ }^{35}$

S. B. Beri, ${ }^{15}$ G. Bernardi, ${ }^{12}$ I. Bertram,${ }^{27}$ A. Besson, ${ }^{9}$ R. Beuselinck,${ }^{28}$ V. A. Bezzubov, ${ }^{26}$ P. C. Bhat,${ }^{36}$ V. Bhatnagar, ${ }^{15}$ M. Bhattacharjee,${ }^{54}$ G. Blazey, ${ }^{38}$ F. Blekman ${ }^{20}$ S. Blessing, ${ }^{35}$ A. Boehnlein,${ }^{36}$ N. I. Bojko, ${ }^{26}$ T. A. Bolton, ${ }^{44}$

F. Borcherding, ${ }^{36}$ K. Bos,${ }^{20}$ T. Bose, ${ }^{52}$ A. Brandt,${ }^{59}$ R. Breedon ${ }^{31}$ G. Briskin,${ }^{58}$ R. Brock,${ }^{50}$ G. Brooijmans,${ }^{36}$ A. Bross, ${ }^{36}$ D. Buchholz, ${ }^{39}$ M. Buehler, ${ }^{37}$ V. Buescher, ${ }^{14}$ V. S. Burtovoi, ${ }^{26}$ J. M. Butler, ${ }^{47}$ F. Canelli, ${ }^{53}$ W. Carvalho, ${ }^{3}$ D. Casey, ${ }^{50}$

Z. Casilum, ${ }^{54}$ H. Castilla-Valdez, ${ }^{19}$ D. Chakraborty, ${ }^{38}$ K. M. Chan, ${ }^{53}$ S. V. Chekulaev, ${ }^{26}$ D. K. Cho, ${ }^{53}$ S. Choi,${ }^{34}$ S. Chopra, ${ }^{55}$ J. H. Christenson, ${ }^{36}$ M. Chung, ${ }^{37}$ D. Claes,${ }^{51}$ A. R. Clark,${ }^{30}$ L. Coney,${ }^{41}$ B. Connolly, ${ }^{35}$ W. E. Cooper, ${ }^{36}$ D. Coppage, ${ }^{43}$

S. Crépé-Renaudin, ${ }^{9}$ M. A. C. Cummings, ${ }^{38}$ D. Cutts, ${ }^{58}$ G. A. Davis, ${ }^{53}$ K. De,${ }^{59}$ S. J. de Jong,${ }^{21}$ M. Demarteau,${ }^{36}$ R. Demina,${ }^{44}$ P. Demine, ${ }^{9}$ D. Denisov,${ }^{36}$ S. P. Denisov, ${ }^{26}$ S. Desai,${ }^{54}$ H. T. Diehl,${ }^{36}$ M. Diesburg, ${ }^{36}$ S. Doulas ${ }^{48}$ Y. Ducros,${ }^{13}$

L. V. Dudko, ${ }^{25}$ S. Duensing,${ }^{21}$ L. Duflot,${ }^{11}$ S. R. Dugad, ${ }^{17}$ A. Duperrin,${ }^{10}$ A. Dyshkant,${ }^{38}$ D. Edmunds,${ }^{50}$ J. Ellison, ${ }^{34}$

J. T. Eltzroth ${ }^{59}$ V. D. Elvira,${ }^{36}$ R. Engelmann,${ }^{54}$ S. Eno, ${ }^{46}$ G. Eppley,${ }^{61}$ P. Ermolov,${ }^{25}$ O. V. Eroshin,${ }^{26}$ J. Estrada,${ }^{53}$ H. Evans,${ }^{52}$ V. N. Evdokimov, ${ }^{26}$ T. Fahland, ${ }^{33}$ D. Fein, ${ }^{29}$ T. Ferbel,,${ }^{53}$ F. Filthaut,${ }^{21}$ H. E. Fisk, ${ }^{36}$ Y. Fisyak,${ }^{55}$ E. Flattum, ${ }^{36}$ F. Fleuret, ${ }^{12}$

M. Fortner,${ }^{38}$ H. Fox,${ }^{39}$ K. C. Frame,${ }^{50}$ S. Fu, ${ }^{52}$ S. Fuess,${ }^{36}$ E. Gallas, ${ }^{36}$ A. N. Galyaev, ${ }^{26}$ M. Gao, ${ }^{52}$ V. Gavrilov, ${ }^{24}$

R. J. Genik II, ${ }^{27}$ K. Genser, ${ }^{36}$ C. E. Gerber, ${ }^{37}$ Y. Gershtein,${ }^{58}$ R. Gilmartin, ${ }^{35}$ G. Ginther, ${ }^{53}$ B. Gómez, ${ }^{5}$ P. I. Goncharov, ${ }^{26}$

J. L. González Solís ${ }^{19}$ H. Gordon ${ }^{55}$ L. T. Goss, ${ }^{60}$ K. Gounder,${ }^{36}$ A. Goussiou, ${ }^{28}$ N. Graf, ${ }^{55}$ P. D. Grannis, ${ }^{54}$

J. A. Green, ${ }^{42}$ H. Greenlee, ${ }^{36}$ Z. D. Greenwood ${ }^{45}$ S. Grinstein, ${ }^{1}$ L. Groer,${ }^{52}$ S. Grünendahl, ${ }^{36}$ A. Gupta, ${ }^{17}$ S. N. Gurzhiev, ${ }^{26}$ G. Gutierrez, ${ }^{36}$ P. Gutierrez, ${ }^{57}$ N. J. Hadley, ${ }^{46}$ H. Haggerty, ${ }^{36}$ S. Hagopian ${ }^{35}$ V. Hagopian, ${ }^{35}$ R. E. Hall ${ }^{32}$ S. Hansen, ${ }^{36}$

J. M. Hauptman, ${ }^{42}$ C. Hays, ${ }^{52}$ C. Hebert, ${ }^{43}$ D. Hedin, ${ }^{38}$ J. M. Heinmiller, ${ }^{37}$ A. P. Heinson, ${ }^{34}$ U. Heintz, ${ }^{47}$ M. D. Hildreth, ${ }^{41}$

R. Hirosky, ${ }^{62}$ J. D. Hobbs,${ }^{54}$ B. Hoeneisen, ${ }^{8}$ Y. Huang,${ }^{49}$ I. Iashvili, ${ }^{34}$ R. Illingworth, ${ }^{28}$ A. S. Ito, ${ }^{36}$ M. Jaffré, ${ }^{11}$

S. Jain, ${ }^{17}$ R. Jesik ${ }^{28}$ K. Johns,${ }^{29}$ M. Johnson, ${ }^{36}$ A. Jonckheere, ${ }^{36}$ H. Jöstlein, ${ }^{36}$ A. Juste, ${ }^{36}$ W. Kahl, ${ }^{44}$ S. Kahn, ${ }^{55}$ E. Kajfasz,${ }^{10}$ A. M. Kalinin, ${ }^{23}$ D. Karmanov, ${ }^{25}$ D. Karmgard,${ }^{41}$ R. Kehoe, ${ }^{50}$ A. Khanov, ${ }^{44}$ A. Kharchilava, ${ }^{41}$ S. K. Kim, ${ }^{18}$ B. Klima, ${ }^{36}$

B. Knuteson, ${ }^{30}$ W. Ko, ${ }^{31}$ J. M. Kohli, ${ }^{15}$ A. V. Kostritskiy, ${ }^{26}$ J. Kotcher, ${ }^{55}$ B. Kothari, ${ }^{52}$ A. V. Kotwal, ${ }^{52}$ A. V. Kozelov, ${ }^{26}$

E. A. Kozlovsky, ${ }^{26}$ J. Krane, ${ }^{42}$ M. R. Krishnaswamy, ${ }^{17}$ P. Krivkova, ${ }^{6}$ S. Krzywdzinski, ${ }^{36}$ M. Kubantsev, ${ }^{44}$ S. Kuleshov, ${ }^{24}$

Y. Kulik, ${ }^{36}$ S. Kunori, ${ }^{46}$ A. Kupco, ${ }^{7}$ V. E. Kuznetsov, ${ }^{34}$ G. Landsberg, ${ }^{58}$ W. M. Lee, ${ }^{35}$ A. Leflat, ${ }^{25}$ C. Leggett, ${ }^{30}$ F. Lehner, ${ }^{36, *}$

C. Leonidopoulos, ${ }^{52}$ J. Li,${ }^{59}$ Q. Z. Li ${ }^{36}$ J. G. R. Lima, ${ }^{3}$ D. Lincoln, ${ }^{36}$ S. L. Linn, ${ }^{35}$ J. Linnemann, ${ }^{50}$ R. Lipton, ${ }^{36}$

A. Lucotte, ${ }^{9}$ L. Lueking, ${ }^{36}$ C. Lundstedt,${ }^{51}$ C. Luo, ${ }^{40}$ A. K. A. Maciel,${ }^{38}$ R. J. Madaras, ${ }^{30}$ V. L. Malyshev, ${ }^{23}$ V. Manankov, ${ }^{25}$ H. S. Mao, ${ }^{4}$ T. Marshall, ${ }^{40}$ M. I. Martin, ${ }^{38}$ A. A. Mayorov, ${ }^{26}$ R. McCarthy,${ }^{54}$ T. McMahon, ${ }^{56}$ H. L. Melanson, ${ }^{36}$

M. Merkin, ${ }^{25}$ K. W. Merritt,${ }^{36}$ C. Miao, ${ }^{58}$ H. Miettinen, ${ }^{61}$ D. Mihalcea,${ }^{38}$ C. S. Mishra,${ }^{36}$ N. Mokhov, ${ }^{36}$ N. K. Mondal,${ }^{17}$ H. E. Montgomery, ${ }^{36}$ R. W. Moore,${ }^{50}$ M. Mostafa, ${ }^{1}$ H. da Motta, ${ }^{2}$ Y. Mutaf,${ }^{54}$ E. Nagy, ${ }^{10}$ F. Nang,${ }^{29}$ M. Narain, ${ }^{47}$

V. S. Narasimham,${ }^{17}$ N. A. Naumann, ${ }^{21}$ H. A. Neal ${ }^{49}$ J. P. Negret, ${ }^{5}$ A. Nomerotski, ${ }^{36}$ T. Nunnemann,${ }^{36}$ D. O'Neil, ${ }^{50}$ V. Oguri, ${ }^{3}$ B. Olivier, ${ }^{12}$ N. Oshima, ${ }^{36}$ P. Padley,${ }^{61}$ L. J. Pan,${ }^{39}$ K. Papageorgiou, ${ }^{37}$ N. Parashar,${ }^{48}$ R. Partridge,${ }^{58}$ N. Parua, ${ }^{54}$

M. Paterno, ${ }^{53}$ A. Patwa, ${ }^{54}$ B. Pawlik, ${ }^{22}$ O. Peters, ${ }^{20}$ P. Pétroff,${ }^{11}$ R. Piegaia, ${ }^{1}$ B. G. Pope, ${ }^{50}$ E. Popkov, ${ }^{47}$ H. B. Prosper,${ }^{35}$

S. Protopopescu ${ }^{55}$ M. B. Przybycien, ${ }^{39, \dagger}$ J. Qian,${ }^{49}$ R. Raja, ${ }^{36}$ S. Rajagopalan, ${ }^{55}$ E. Ramberg, ${ }^{36}$ P. A. Rapidis, ${ }^{36}$

N. W. Reay ${ }^{44}$ S. Reucroft,${ }^{48}$ M. Ridel,${ }^{11}$ M. Rijssenbeek,${ }^{54}$ F. Rizatdinova, ${ }^{44}$ T. Rockwell,${ }^{50}$ M. Roco, ${ }^{36}$ C. Royon, ${ }^{13}$

P. Rubinov, ${ }^{36}$ R. Ruchti, ${ }^{41}$ J. Rutherfoord, ${ }^{29}$ B. M. Sabirov, ${ }^{23}$ G. Sajot, ${ }^{9}$ A. Santoro, ${ }^{3}$ L. Sawyer, ${ }^{45}$ R. D. Schamberger,${ }^{54}$

H. Schellman, ${ }^{39}$ A. Schwartzman, ${ }^{1}$ N. Sen, ${ }^{61}$ E. Shabalina, ${ }^{37}$ R. K. Shivpuri, ${ }^{16}$ D. Shpakov,${ }^{48}$ M. Shupe,${ }^{29}$

R. A. Sidwell, ${ }^{44}$ V. Simak, ${ }^{7}$ H. Singh, ${ }^{34}$ V. Sirotenko, ${ }^{36}$ P. Slattery,${ }^{53}$ E. Smith, ${ }^{57}$ R. P. Smith,${ }^{36}$ R. Snihur, ${ }^{39}$ G. R. Snow, ${ }^{51}$

J. Snow, ${ }^{56}$ S. Snyder,${ }^{55}$ J. Solomon, ${ }^{37}$ Y. Song, ${ }^{59}$ V. Sorín, ${ }^{1}$ M. Sosebee,${ }^{59}$ N. Sotnikova, ${ }^{25}$ K. Soustruznik, ${ }^{6}$ M. Souza, ${ }^{2}$

N. R. Stanton, ${ }^{44}$ G. Steinbrück ${ }^{52}$ R. W. Stephens, ${ }^{59}$ D. Stoker, ${ }^{33}$ V. Stolin, ${ }^{24}$ A. Stone,${ }^{45}$ D. A. Stoyanova, ${ }^{26}$ M. A. Strang, ${ }^{59}$

M. Strauss,${ }^{57}$ M. Strovink, ${ }^{30}$ L. Stutte,${ }^{36}$ A. Sznajder, ${ }^{3}$ M. Talby,${ }^{10}$ W. Taylor, ${ }^{54}$ S. Tentindo-Repond ${ }^{35}$ S. M. Tripathi, ${ }^{31}$

T. G. Trippe,${ }^{30}$ A. S. Turcot, ${ }^{55}$ P. M. Tuts, ${ }^{52}$ V. Vaniev, ${ }^{26}$ R. Van Kooten, ${ }^{40}$ N. Varelas, ${ }^{37}$ L. S. Vertogradov, ${ }^{23}$

F. Villeneuve-Seguier, ${ }^{10}$ A. A. Volkov, ${ }^{26}$ A. P. Vorobiev, ${ }^{26}$ H. D. Wahl,${ }^{35}$ H. Wang, ${ }^{39}$ Z.-M. Wang, ${ }^{54}$ J. Warchol, ${ }^{41}$ G. Watts,${ }^{63}$

M. Wayne ${ }^{41}$ H. Weerts, ${ }^{50}$ A. White, ${ }^{59}$ J. T. White, ${ }^{60}$ D. Whiteson, ${ }^{30}$ D. A. Wijngaarden, ${ }^{21}$ S. Willis, ${ }^{38}$ S. J. Wimpenny,${ }^{34}$

J. Womersley, ${ }^{36}$ D. R. Wood, ${ }^{48}$ Q. Xu,${ }^{49}$ R. Yamada, ${ }^{36}$ P. Yamin,${ }^{55}$ T. Yasuda, ${ }^{36}$ Y. A. Yatsunenko, ${ }^{23}$ K. Yip, ${ }^{55}$

S. Youssef, ${ }^{35}$ J. Yu, ${ }^{59}$ M. Zanabria, ${ }^{5}$ X. Zhang, ${ }^{57}$ H. Zheng, ${ }^{41}$ B. Zhou ${ }^{49}$ Z. Zhou, ${ }^{42}$ M. Zielinski, ${ }^{53}$ D. Zieminska, ${ }^{40}$ A. Zieminski, ${ }^{40}$ V. Zutshi, ${ }^{38}$ E. G. Zverev, ${ }^{25}$ and A. Zylberstejn ${ }^{13}$

\footnotetext{
(DØ Collaboration)

${ }^{1}$ Universidad de Buenos Aires, Buenos Aires, Argentina

${ }^{2}$ LAFEX, Centro Brasileiro de Pesquisas Físicas, Rio de Janeiro, Brazil

${ }^{3}$ Universidade do Estado do Rio de Janeiro, Rio de Janeiro, Brazil

${ }^{4}$ Institute of High Energy Physics, Beijing, People's Republic of China

${ }^{5}$ Universidad de los Andes, Bogotá, Colombia
} 
${ }^{6}$ Charles University, Center for Particle Physics, Prague, Czech Republic

${ }^{7}$ Institute of Physics, Academy of Sciences, Center for Particle Physics, Prague, Czech Republic

${ }^{8}$ Universidad San Francisco de Quito, Quito, Ecuador

${ }^{9}$ Institut des Sciences Nucléaires, IN2P3-CNRS, Universite de Grenoble 1, Grenoble, France

${ }^{10}$ CPPM, IN2P3-CNRS, Université de la Méditerranée, Marseille, France

${ }^{11}$ Laboratoire de l'Accélérateur Linéaire, IN2P3-CNRS, Orsay, France

${ }^{12}$ LPNHE, Universités Paris VI and VII, IN2P3-CNRS, Paris, France

${ }^{13}$ DAPNIA/Service de Physique des Particules, CEA, Saclay, France

${ }^{14}$ Universität Mainz, Institut für Physik, Mainz, Germany

${ }^{15}$ Panjab University, Chandigarh, India

${ }^{16}$ Delhi University, Delhi, India

${ }^{17}$ Tata Institute of Fundamental Research, Mumbai, India

${ }^{18}$ Seoul National University, Seoul, Korea

${ }^{19}$ CINVESTAV, Mexico City, Mexico

${ }^{20}$ FOM-Institute NIKHEF and University of Amsterdam/NIKHEF, Amsterdam, The Netherlands

${ }^{21}$ University of Nijmegen/NIKHEF, Nijmegen, The Netherlands

${ }^{22}$ Institute of Nuclear Physics, Kraków, Poland

${ }^{23}$ Joint Institute for Nuclear Research, Dubna, Russia

${ }^{24}$ Institute for Theoretical and Experimental Physics, Moscow, Russia

${ }^{25}$ Moscow State University, Moscow, Russia

${ }^{26}$ Institute for High Energy Physics, Protvino, Russia

${ }^{27}$ Lancaster University, Lancaster, United Kingdom

${ }^{28}$ Imperial College, London, United Kingdom

${ }^{29}$ University of Arizona, Tucson, Arizona 85721

${ }^{30}$ Lawrence Berkeley National Laboratory and University of California, Berkeley, California 94720

${ }^{31}$ University of California, Davis, California 95616

${ }^{32}$ California State University, Fresno, California 93740

${ }^{33}$ University of California, Irvine, California 92697

${ }^{34}$ University of California, Riverside, California 92521

${ }^{35}$ Florida State University, Tallahassee, Florida 32306

${ }^{36}$ Fermi National Accelerator Laboratory, Batavia, Illinois 60510

${ }^{37}$ University of Illinois at Chicago, Chicago, Illinois 60607

${ }^{38}$ Northern Illinois University, DeKalb, Illinois 60115

${ }^{39}$ Northwestern University, Evanston, Illinois 60208

${ }^{40}$ Indiana University, Bloomington, Indiana 47405

${ }^{41}$ University of Notre Dame, Notre Dame, Indiana 46556

${ }^{42}$ Iowa State University, Ames, Iowa 50011

${ }^{43}$ University of Kansas, Lawrence, Kansas 66045

${ }^{44}$ Kansas State University, Manhattan, Kansas 66506

${ }^{45}$ Louisiana Tech University, Ruston, Louisiana 71272

${ }^{46}$ University of Maryland, College Park, Maryland 20742

${ }^{47}$ Boston University, Boston, Massachusetts 02215

${ }^{48}$ Northeastern University, Boston, Massachusetts 02115

${ }^{49}$ University of Michigan, Ann Arbor, Michigan 48109

${ }^{50}$ Michigan State University, East Lansing, Michigan 48824

${ }^{51}$ University of Nebraska, Lincoln, Nebraska 68588

${ }^{52}$ Columbia University, New York, New York 10027

${ }^{53}$ University of Rochester, Rochester, New York 14627

${ }^{54}$ State University of New York, Stony Brook, New York 11794

${ }^{55}$ Brookhaven National Laboratory, Upton, New York 11973

${ }^{56}$ Langston University, Langston, Oklahoma 73050

${ }^{57}$ University of Oklahoma, Norman, Oklahoma 73019

${ }^{58}$ Brown University, Providence, Rhode Island 02912

${ }^{59}$ University of Texas, Arlington, Texas 76019

${ }^{60}$ Texas A\&M University, College Station, Texas 77843

${ }^{61}$ Rice University, Houston, Texas 77005

${ }^{62}$ University of Virginia, Charlottesville, Virginia 22901

${ }^{63}$ University of Washington, Seattle, Washington 98195 
(Received 8 April 2002; published 29 August 2002)

Based on $85 \mathrm{pb}^{-1}$ data of $p \bar{p}$ collisions at $\sqrt{s}=1.8 \mathrm{TeV}$ collected using the $\mathrm{D} \emptyset$ detector at Fermilab during the 1994-1995 run of the Tevatron, we present a direct measurement of the total decay width of the $W$ boson $\Gamma_{W}$. The width is determined from the transverse mass spectrum in the $W \rightarrow e+\nu_{e}$ decay channel and found to be $\Gamma_{W}=2.23_{-0.14}^{+0.15}$ (stat) \pm 0.10 (syst) $\mathrm{GeV}$, consistent with the expectation from the standard model.

DOI: 10.1103/PhysRevD.66.032008

PACS number(s): 13.38.Be, 14.70.Fm

\section{INTRODUCTION}

The theory that describes the fundamental particle interactions is called the standard model (SM). The standard model is a gauge field theory that comprises the GlashowWeinberg-Salam (GWS) model [1-3] of the weak and electromagnetic interactions and quantum chromodynamics (QCD) [4-6], the theory of the strong interactions. The discovery of the $W[7,8]$ and $Z[9,10]$ bosons in 1983 by the UA1 and UA2 Collaborations at the CERN $p \bar{p}$ collider provided a direct confirmation of the unification of the weak and electromagnetic interactions. Experiments have been refining the measurements of the characteristics of the $W$ and $Z$ bosons. The total decay width of $W$ boson, $\Gamma_{W}$, is given in the SM in terms of the masses of the gauge bosons and their couplings to their decay products.

In $p \bar{p}$ collisions, $W$ bosons are produced by processes of the type $u \bar{d}$ or $\bar{u} d \rightarrow W$, followed by subsequent leptonic or hadronic decay: $W \rightarrow \ell \nu$ or $W \rightarrow q^{\prime} \bar{q}$, where $\ell=e, \mu, \tau$, and $q^{\prime}$ or $q$ represent one of the quarks $u, d, c, s$, or $b$ (but not $t$ since top quark is heavier than the $W$ boson).

At lowest order in perturbation theory, the SM predicts the partial decay width $\Gamma(W \rightarrow e \nu)$ of $W \rightarrow e \nu$ to be $\Gamma(W$ $\rightarrow e \nu)=g^{2} M_{W} / 48 \pi$ [11]. Including radiative corrections, this can be rewritten as

$$
\Gamma(W \rightarrow e \nu)=\frac{G_{F} M_{W}^{3}}{6 \sqrt{2} \pi}\left(1+\delta_{\mathrm{SM}}\right),
$$

where $G_{F} / \sqrt{2}=g^{2} / 8 M_{W}^{2}, g$ is the charged current coupling, and $M_{W}$ is the mass of the $W$ boson. The SM radiative correction $\delta_{\mathrm{SM}}$ is calculated [12] to be less than $\frac{1}{2} \%$. By using the experimental values of $G_{F}$ (measured from muon decay [13]) and $M_{W}$ (measured at the Fermilab Tevatron collider $[14,15]$ and CERN $e^{+} e^{-}$collider LEP2 [16-19]), the predicted partial width is [11] $\Gamma(W \rightarrow e \nu)=226.5 \pm 0.3 \mathrm{MeV}$.

A $W$ boson has three leptonic decay channels and two dominant hadronic decay channels $W \rightarrow e \bar{\nu}, \mu \bar{\nu}, \tau \bar{\nu}$, and $q q^{\prime}$, where $q$ is $u$ or $c$, and $q^{\prime}$ is the appropriate CabibboKobayashi-Maskawa (CKM) mixture of $d$ and $s$. Other hadronic decay channels are greatly suppressed by CKM offdiagonal matrix elements. Considering the three color charges for quarks, these nine leptonic and hadronic channels yield a total width of $\approx 9 \Gamma(W \rightarrow e \nu)$. Including QCD corrections, the leptonic decay branching ratio is $B(W \rightarrow e \nu)$

\footnotetext{
*Also at University of Zurich, Zurich, Switzerland.

${ }^{\dagger}$ Also at Institute of Nuclear Physics, Krakow, Poland.
}

$=1 /\left\{3+6\left[1+\alpha_{s}\left(M_{W}\right) / \pi+\mathcal{O}\left(\alpha_{s}^{2}\right)\right]\right\}$, leading to the SM prediction for the full width of the $W$ boson [11] of $\Gamma_{W}$ $=2.0921 \pm 0.0025 \mathrm{GeV}$.

Historically, the accurate determination of the width of the $W$ boson was available through an indirect measurement using the ratio $\mathcal{R}$ of the $W \rightarrow e \nu$ and $Z \rightarrow e e$ cross sections

$$
\begin{aligned}
\mathcal{R} & =\frac{\sigma(p \bar{p} \rightarrow W+X) \cdot \operatorname{Br}(W \rightarrow e \nu)}{\sigma(p \bar{p} \rightarrow Z+X) \cdot \operatorname{Br}(Z \rightarrow e e)} \\
& =\frac{\sigma_{W}}{\sigma_{Z}} \cdot \frac{\operatorname{Br}(W \rightarrow e \nu)}{\operatorname{Br}(Z \rightarrow e e)} .
\end{aligned}
$$

A measurement of $\mathcal{R}$, together with a calculation [20] of the ratio of production cross sections $\sigma_{W} / \sigma_{Z}$ and the measurement of the branching faction $\operatorname{Br}(Z \rightarrow e e)=\Gamma(Z$ $\rightarrow e e) / \Gamma(Z)$ from the CERN $e^{+} e^{-}$collider (LEP) [21], can be used to extract the $W$ boson leptonic branching ratio $\operatorname{Br}(W \rightarrow e \nu)=\Gamma(W \rightarrow e \nu) / \Gamma(W)$, which, in turn, yields the full width of the $W$ boson from calculated partial decay width $\Gamma(W \rightarrow e \nu)$. Thus, in this indirect measurement, calculations of $\sigma_{W} / \sigma_{Z}$ and the partial width $\Gamma(W \rightarrow e \nu)$ yield $\Gamma_{W}$ in the context of the SM. This method was first used by the UA1 [22] and UA2 [23] Collaborations. More recently, the CDF [24] and DØ [25] Collaborations obtained $\Gamma_{W}$ $=2.064 \pm 0.084 \mathrm{GeV}$ and $\Gamma_{W}=2.169 \pm 0.079 \mathrm{GeV}$, respectively, using this technique.

The value of $\Gamma_{W}$ can also be obtained from the line shape of the transverse mass $m_{T}$ of the $W$ boson, because the BreitWigner (width) component of the line shape falls off more slowly at high $m_{T}$ than the resolution component does [12]. The transverse mass is given by

$$
m_{T}=\sqrt{2 E_{T}^{e} E_{T}^{\nu}\left[1-\cos \left(\phi^{e}-\phi^{\nu}\right)\right]},
$$

where $E_{T}^{e}$ and $E_{T}^{\nu}$ are the transverse energies, and $\phi^{e}$ and $\phi^{\nu}$ are the azimuthal angles of the electron and neutrino, respectively. The transverse mass has a kinematic upper limit at the value of $M_{W}$, and the shape of the $m_{T}$ distribution at this upper limit, called the "Jacobian edge," is sensitive to $\Gamma_{W}$ [26]. Using this technique, the Collider Detector at Fermilab (CDF) Collaboration reported [27] a measurement of $\Gamma_{W}$ $=2.05 \pm 0.10$ (stat) \pm 0.08 (syst) GeV. Figure 1 shows the $m_{T}$ spectrum shape expected for different values of $\Gamma_{W}$ and indicates the sensitivity of the tail of the transverse mass distribution to $\Gamma_{W}$. Clearly, the effect is greatest in the region above $m_{W}$.

The direct measurement of $\Gamma_{W}$ complements the indirect measurement through $\mathcal{R}$ in several ways: theoretical inputs for $\sigma_{W} / \sigma_{Z}$ and $\Gamma(W \rightarrow e \nu)$, which may be sensitive to non-SM coupling of the $W$ boson, are not needed; the direct 


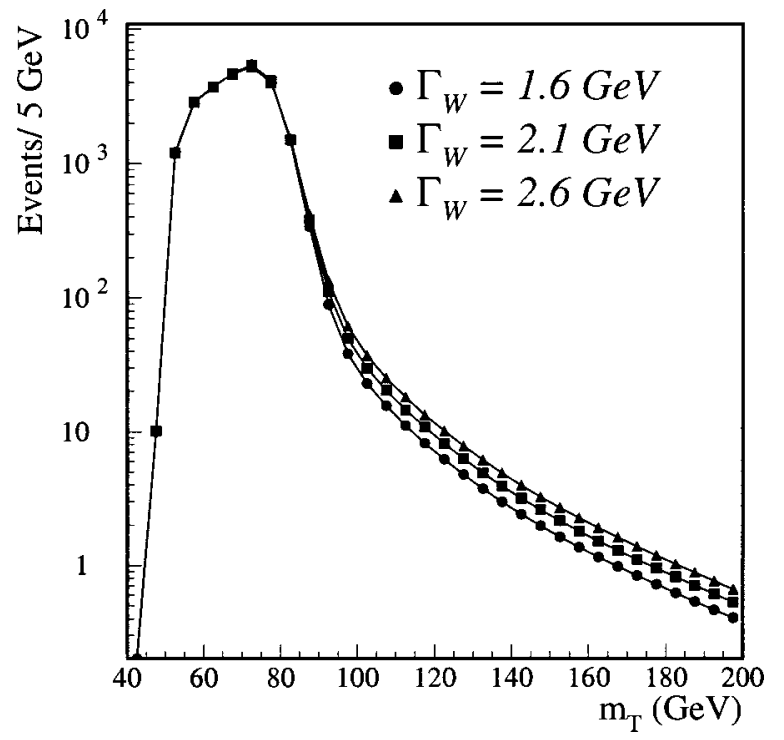

FIG. 1. Monte Carlo simulations of the transverse mass spectrum for different $W$ boson widths. The selections $E_{T}(e)>25 \mathrm{GeV}$ and $E_{T}(\nu)>25 \mathrm{GeV}$, are applied to MC sample. The circles show the spectrum for $\Gamma_{W}=1.60 \mathrm{GeV}$, the squares for $\Gamma_{W}=2.10 \mathrm{GeV}$, and triangles for $\Gamma_{W}=2.60 \mathrm{GeV}$. Distributions are normalized arbitrarily in the transverse mass region shown.

measurement explores the region above the $W$ boson mass pole, where possible new phenomena such as an additional heavy vector boson $\left(W^{\prime}\right)$ can contribute; it is desirable to have more than one method of measuring a given property. The sources of systematic errors in the two methods are different, and the direct method will be important when the measurement through $\mathcal{R}$ becomes limited by systematic uncertainty.

The paper is organized as follows. In Sec. II, we give a brief description of the $\mathrm{D} \emptyset$ detector. Particle identification and event selection are discussed in Sec. III. The analysis procedure, including background estimation and Monte Carlo simulation, is described in Sec. IV, and the conclusions are presented in Sec. V. For more detailed information on this analysis, see Ref. [28].

\section{THE DØ DETECTOR}

\section{A. Experimental apparatus}

The D $\varnothing$ detector [30] comprises three major systems. The innermost of these is a nonmagnetic tracker used in the reconstruction of charged particle tracks. The tracker is surrounded by central and forward uranium/liquid-argon sampling calorimeters. These calorimeters are used to identify electrons, photons, and hadronic jets, and to reconstruct their energies. The calorimeters are surrounded by a muon spectrometer used in the identification of muons and the reconstruction of their momenta. We use a coordinate system $(\rho, \theta$, $\phi)$ where $\rho$ is the perpendicular distance from the beam line, $\theta$ is the polar angle measured relative to the proton beam direction $z$, and $\phi$ is the azimuthal angle. The pseudorapidity $\eta$ is defined as $-\ln (\tan \theta / 2)$. For this analysis, the relevant components are the tracking system and the calorimeters.
The central tracking system provides a measurement of the energy loss due to ionization $(d E / d x)$ for tracks within its tracking volume. This information is used to help distinguish prompt electrons from $e^{+} e^{-}$pairs due to photon conversions.

The structure of the calorimeter has been optimized to distinguish electrons and photons from hadrons and to measure their energies. It is composed of three sections: the central calorimeter (CC), and two end calorimeters (EC). The $\eta$ coverage for electrons used in this analysis is $|\eta|<1.1$ [29] in the $\mathrm{CC}$ region, which consists of $32 \phi$ modules. The calorimeter is segmented longitudinally into three sections, the electromagnetic (EM) calorimeter, the fine hadronic (FH) calorimeter, and the coarse hadronic $(\mathrm{CH})$ calorimeter. The EM calorimeter is subdivided longitudinally into four layers (EM1-EM4). The first, second and fourth layers of the EM calorimeter are transversely divided into cells of size $\Delta \eta$ $\times \Delta \phi=0.1 \times 0.1$. The electromagnetic shower maximum occurs in the third layer, which is divided into finer units of $0.05 \times 0.05$ to improve the measurement of the shower shape and spatial resolution. There are $16 \mathrm{FH}$ modules and $16 \mathrm{CH}$ modules in $\phi$. The fine hadronic calorimeter is subdivided longitudinally into three fine hadronic layers (FH1-FH3), and there is only one coarse hadronic layer.

\section{B. Trigger}

The $\mathrm{D} \emptyset$ trigger has three levels, each applying increasingly more sophisticated selection criteria to an event. The lowest level trigger, level 0 , uses scintillation counters located on the inner faces of the forward calorimeters to signal the presence of an inelastic $p \bar{p}$ collision. Data from the level 0 counters, the calorimeter, and the muon chambers are sent to the level 1 trigger, which provides a trigger on total transverse energy $\left(E_{T}\right)$, missing transverse energy $\left(\boldsymbol{E}_{T}\right), E_{T}$ of individual calorimeter towers, and/or the presence of a muon. These triggers operate in less than $3.5 \mu \mathrm{s}$, the time between bunch crossings. Some calorimeter and muon-based triggers require additional time, which is provided by a level 1.5 trigger system.

Level 1 (and 1.5) triggers initiate a level 2 trigger system that consists of a farm of microprocessors. These microprocessors run simplified versions of the off-line event reconstruction algorithms to select events of interest.

\section{PARTICLE IDENTIFICATION AND EVENT SELECTION}

This analysis relies on the $\mathrm{D} \varnothing$ detector's ability to identify electrons and neutrinos which is associated with the undetected energy. We use both $W \rightarrow e \nu$ and $Z \rightarrow e^{+} e^{-}$candidate samples for this analysis. The $W$ boson candidate sample provides the signal events, while the $Z \rightarrow e^{+} e^{-}$candidate sample is used to calibrate both the data and the Monte Carlo (MC) simulation. Candidate $W$ and $Z$ events are identified by the presence of an electron and a neutrino, or by the presence of two electrons with an invariant mass consistent with the mass of the $Z$ boson, respectively. Electrons from $W$ and $Z$ boson decays typically have large transverse 
energy and are isolated from other particles. They are associated with a track in the tracking system and with a large deposit of energy in one of the EM calorimeters. Neutrinos do not interact in the detector, and thus create an apparent transverse energy imbalance in an event. For each $W$ boson candidate event, we measure the energy imbalance in the plane transverse to the beam direction $\left(\boldsymbol{E}_{T}\right)$, and attribute this to the neutrino. The following sections provide a brief summary of the procedure [25] used in this analysis.

\section{A. Electron identification}

Identification of electrons starts at the trigger level with the selection of clusters of electromagnetic energy. At level 1 , the trigger searches for EM calorimeter towers $(\Delta \phi$ $\times \Delta \eta=0.2 \times 0.2$ ) with signals that exceed predefined thresholds. $W$ boson triggers require that the energy deposited in a single EM calorimeter tower exceed $10 \mathrm{GeV}$. Those events that satisfy the level 1 trigger are processed by the level 2 filter. The trigger towers are combined with the energy in the surrounding calorimeter cells within a window of $\Delta \phi \times \Delta \eta$ $=0.6 \times 0.6$.

Events are selected at level 2 if the transverse energy in this window exceeds $20 \mathrm{GeV}$. In addition to the $E_{T}$ requirement, the longitudinal and transverse shower shapes are required to match those expected for electromagnetic showers. The longitudinal shower shape is described by the fraction of the energy deposited in each of the four EM layers of the calorimeter. The transverse shower shape is characterized by energy deposition patterns in the third EM layer. The difference between the energies in concentric regions covering $0.25 \times 0.25$ and $0.15 \times 0.15$ in $\Delta \eta \times \Delta \phi$ must be consistent with that expected for an electron [30].

In addition, the electron candidates are required to deposit at least $90 \%$ of their total calorimetric energy in the EM section and to be isolated from other calorimetric energy deposits, which is $f_{\mathrm{EM}}=E_{\mathrm{EM}} / E_{\text {total }}>0.9$. To be considered isolated, electrons must satisfy the isolation requirement $f_{\text {iso }}<0.15$, where $f_{\text {iso }}$ is defined as

$$
f_{\text {iso }}=\frac{E_{\text {total }}(0.4)-E_{\mathrm{EM}}(0.2)}{E_{\mathrm{EM}}(0.2)}
$$

in which $E_{\text {total }}(0.4)$ is the total energy and $E_{\mathrm{EM}}(0.2)$ the electromagnetic energy, in cones of radius $R=\sqrt{(\Delta \eta)^{2}+(\Delta \phi)^{2}}$ $=0.4$ and 0.2 , respectively. This enhances the signal expected from isolated electrons in $W$ and $Z$ boson decay.

After events are selected with isolated electromagnetic showers at the on-line trigger level, we apply the offline selection to these showers. For the purpose to study the background, we first define "loose" electron. Those EM clusters are require to locate within the sensitive area of a calorimeter module, have an associated track in the central tracking volume and $|\eta|<1.1$. To avoid areas of reduced response between neighboring calorimeter modules, the azimuthal angle of electrons is required to be at least $\Delta \phi=0.10 \times 2 \pi / 32$ radians away from the position of a module boundary. We further impose a set of off-line tighter criteria to identify electrons, thereby reducing the background from QCD mul- tijet events. The first step in identifying an electron is to form a cluster around the trigger tower using a nearest neighbor algorithm. As at the trigger level, the cluster is required to be isolated $\left(f_{\text {iso }}<0.15\right)$. To increase the likelihood that the cluster is due to an electron and not a photon, a charged track from the central tracking system is required to point to the center of the EM cluster. We extrapolate the track to the third EM layer of the calorimeter and calculate the distance between the extrapolated track and the cluster centroid along the azimuthal direction $(\rho \Delta \phi)$ and in the $z$ direction $(\Delta z)$. The position of cluster centroid is defined at the radius of the third EM layer of the calorimeter. This position of the EM cluster is connected to the associated one in the central tracking system and extrapolated to the beam line, which defines the $z$ position of the event vertex. The electron $E_{T}$ is calculated using this vertex definition [25]. The variable

$$
\sigma_{\mathrm{trk}}^{2}=\left(\frac{\rho \Delta \phi}{\sigma_{\rho \phi}}\right)^{2}+\left(\frac{\Delta z}{\sigma_{z}}\right)^{2}
$$

where $\sigma_{\rho \phi}$ and $\sigma_{z}$ are the respective track resolutions, quantifies the quality of the match. A requirement of $\sigma_{\text {trk }}<5$ is imposed on the data. These clusters are then subjected to a four-variable likelihood test $[31,32]$. The four variables are the following.

A $\chi^{2}$ comparison of the shower shape with the expected shape of an electromagnetic shower, computed using a 41variable covariance matrix [33] for the energy depositions in the cells of the electromagnetic calorimeter and the location of event vertex.

The electromagnetic energy fraction, defined as the ratio of shower energy in the EM section of the calorimeter relative to the sum of EM energy plus the energy in the first hadronic section of the calorimeter.

A comparison of the track position to the position of cluster centroid, as defined in Eq. (5).

The ionization, $d E / d x$, along the track. This is used to reduce contamination due to $e^{+} e^{-}$pairs from photon conversions, mainly from jets fragmenting into neutral pions. The $e^{+} e^{-}$pair from photon conversion has a double value of $d E / d x$ for a genuine electron due to two overlapping tracks.

To good approximation, these four variables are independent of each other for electron showers. Electrons that satisfy all above criteria are called "tight" electrons.

Electron energies are corrected for the underlying event energy that enter into the electron windows. The electromagnetic energy scale is determined in the test beam data, and adjusted to make the peak of the $Z \rightarrow e^{+} e^{-}$invariant mass agree with the known mass of the $Z$ boson [21]. We found it to be $0.9545 \pm 0.0008$. The electron energy scale is discussed in detail in Ref. [15].

\section{B. Missing transverse energy}

The primary sources of missing energy in an event include the neutrinos that pass through the calorimeter undetected and the calorimeter resolution. The energy imbalance is measured only in the transverse plane because of the lost particles emitted at small angles (within the beam pipes). The 


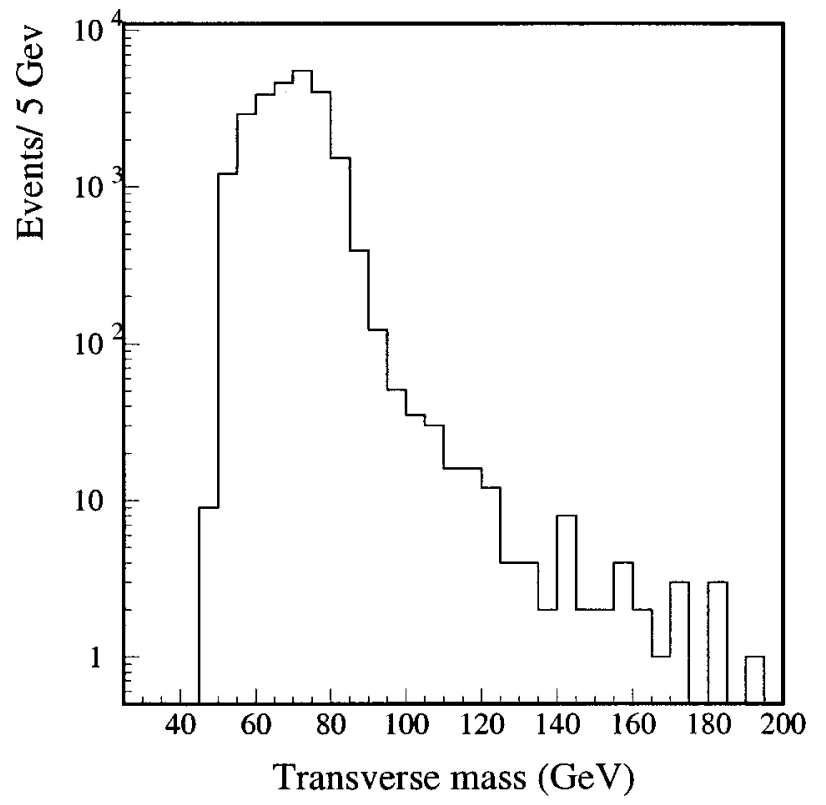

FIG. 2. Transverse mass distribution of $W \rightarrow e \nu$ event candidates.

missing transverse energy is calculated by taking the negative of the vector sum of the transverse energy in all of the calorimeter cells. This gives both the magnitude and direction of $\boldsymbol{E}_{T}$, allowing the calculation of the transverse mass of the $W$ boson candidates.

\section{Event selection}

The $W$ boson data sample used in this analysis was collected during the 1994-1995 run of the Fermilab Tevatron collider, and corresponds to an integrated luminosity of $85.0 \pm 3.6 \mathrm{pb}^{-1}$. Events are selected by requiring one tight electron in the central calorimeter $(|\eta|<1.1)$ [29] with $E_{T}$ $>25 \mathrm{GeV}$. In addition, events are required to have $\mathbb{E}_{T}$ $>25 \mathrm{GeV}$ and $W$ transverse momentum $p_{T}(W)<15 \mathrm{GeV}$, which is combined transverse momentum of electron and $\mathbb{E}_{T}$ (neutrino). After applying all of the described selections, a total of $24487 \mathrm{~W}$ boson candidates is selected. There are 24479 candidates in the region $0-200 \mathrm{GeV}$, while 8(2) candidates have $m_{T}>200(250) \mathrm{GeV}$. Figure 2 shows the transverse mass distribution of the $W \rightarrow e \nu$ candidates.

Candidates for the process $Z \rightarrow e^{+} e^{-}$are required to have two tight electrons, each with $E_{T}>25 \mathrm{GeV}$ in the CC. The invariant mass of the dielectron pair is required to satisfy $60 \mathrm{GeV}<m_{e e}<120 \mathrm{GeV}$. A total of $1997 \mathrm{Z}$ boson candidates is selected. Figure 3 shows the invariant mass distribution of the $Z \rightarrow e^{+} e^{-}$candidates.

\section{ANALYSIS PROCEDURE}

In this section, we describe the Monte Carlo simulation program used to model the transverse mass spectrum. The background from the dominant processes that can mimic the $W \rightarrow e \nu$ signal is also estimated. We compare the data with the expectation from the Monte Carlo simulation and extract

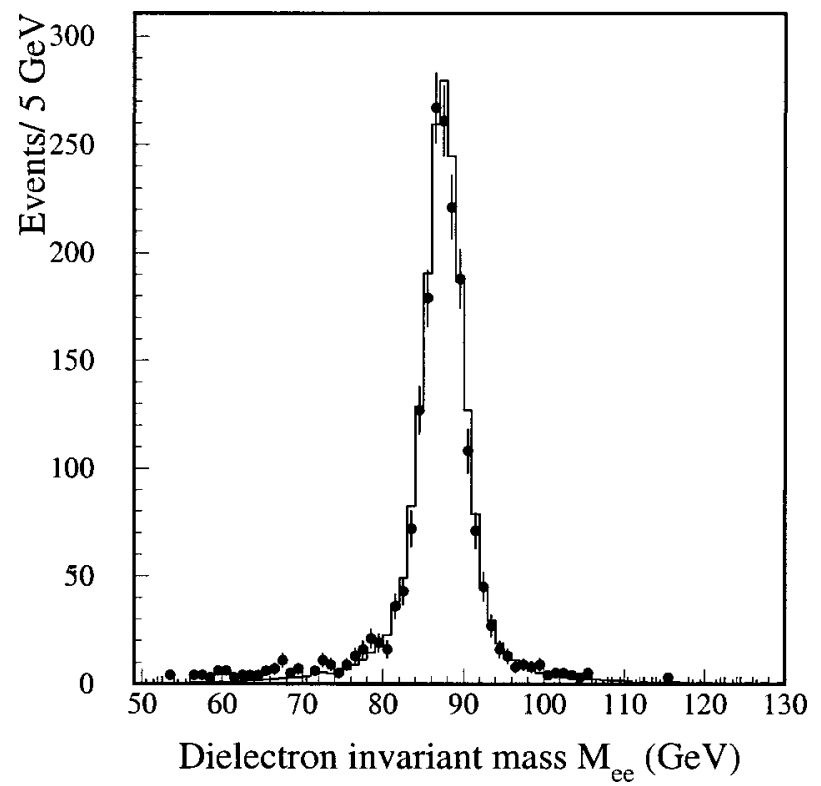

FIG. 3. Invariant mass distribution of $Z \rightarrow e^{+} e^{-}$events compared to Monte Carlo simulation. The histogram is the MC and the black dot with error bar is the data. The $Z \rightarrow e^{+} e^{-}$candidates require both electrons be in the $\mathrm{CC}$.

the decay width of the $W$ boson using log-likelihood fits to the $W$ boson transverse mass distribution.

\section{A. Monte Carlo simulation}

We use the same Monte Carlo program for the earlier $W$ boson mass measurement $[15,34,35]$. The transverse mass spectrum for the $W$ boson is modeled in three steps: $W$ boson production, $W$ boson decay, and a parametrized detector simulation.

We first simulate the production of the $W$ boson by generating its four momentum and other event characteristics, such as the $z$ position of the interaction vertex and the run luminosity. The luminosity is used to parametrize luminosity-dependent effects. The full cross section depends on the mass, pseudorapidity, and transverse momentum of $W$ boson. The dependence of pseudorapidity and transverse momentum are correlated. We use RESBOS [36] to calculate the dependence and use it as input to our MC program. To lowest-order, the mass dependence of the $W$ boson production follows the Breit-Wigner distribution

$$
\sigma(Q)=\mathcal{L}_{q \bar{q}}(Q) \frac{Q^{2}}{\left(Q^{2}-M_{W}^{2}\right)^{2}+Q^{4} \Gamma_{W}^{2} / M_{W}^{2}},
$$

where $Q$ is the invariant mass of $W$ boson, $M_{W}$ is the pole mass and $\Gamma_{W}$ the decay width of the $W$ boson, and $\mathcal{L}_{q \bar{q}}(Q)$ is called the parton luminosity. To evaluate $\mathcal{L}_{q \bar{q}}(Q)$, we generate $W \rightarrow e \nu$ events using the leading-order RESBOS event generator and the different PDF models described in Refs. $[37,38]$. The events are then selected using the same kinematic and fiducial constrains as for the $W$ and $Z$ boson data samples. The resulting event distribution is proportional to the parton luminosity, which we parametrize with the function [39]: 


$$
\mathcal{L}_{q \bar{q}}(Q)=\frac{e^{-\beta Q}}{Q},
$$

where $\beta$ is obtained from a fit of the $\mathrm{MC}$ events to Eq. (6).

The decay of the $W$ boson is simulated in the $\mathrm{MC}$ and used to calculate the transverse momentum of the electron and other decay products. Any radiation from the decay electron or from the $W$ boson can bias the measurement and has to be taken into account. $W \rightarrow \tau \nu \rightarrow e \nu \bar{\nu} \bar{\nu}$ events are indistinguishable from $W \rightarrow e \nu$ and are also included in the model, using a branching ratio of $\operatorname{Br}(\tau \rightarrow e \nu \bar{\nu}) /[1+\operatorname{Br}(\tau \rightarrow e \nu \bar{\nu})]$ $=0.151$.

Finally, we apply a parametrized detector simulation to the momenta of all decay products to simulate any observed recoil jets and electron momenta. The parameters giving the electron and recoil system response of the detector are fixed using data, which include $Z$ bosons and their recoil jets, to study calorimeter response and resolution. The response to jets and electrons is parametrized as a function of energy and angle. Also included in the detector parametrization are effects due to the longitudinal spread of the interaction vertex and the luminosity-dependent response of the detector caused by multiple collisions. After detector simulation of MC $W$ events, we apply the same event selections of $W$ $\rightarrow e \nu$ data to the MC sample.

Uncertainties in the input parameters to the MC will eventually limit the accuracy of the width measurement of the $W$ boson. To study the uncertainties, we allow these input parameters to vary by one standard deviation and regenerate the corresponding transverse mass spectrum. We then fit it with a nominal MC template. If the positive and negative variations of the width of the $W$ boson with respect to a parameter are not symmetric, the larger value is used for the uncertainty. This estimation is used to estimate the impact of the electron energy resolution, hadronic energy resolution, electron energy scale, hadronic energy scale, dependence on the $W$ boson mass, electron angular calibration, and radiative corrections. Detailed studies of these parameters can be found in Ref. [15]. The uncertainties on $\Gamma_{W}$ from the electron energy resolution and scale are 27 and $41 \mathrm{MeV}$, respectively. The uncertainties from the hadronic energy resolution and scale lead to variations in $\Gamma_{W}$ of 55 and $22 \mathrm{MeV}$, respectively. The error on the $W$ boson mass of $37 \mathrm{MeV}$, which is the uncertainty of world average of $W$ mass $m_{W}=80.436$ $\pm 0.037 \mathrm{GeV}$, has an effect of $15 \mathrm{MeV}$ on $\Gamma_{W}$. The uncertainties from radiative decay and electron angular calibration correspond to 10 and $9 \mathrm{MeV}$, respectively.

Uncertainties on $\Gamma_{W}$ also arise from uncertainties in the production model and the parton distribution functions (PDF's). The uncertainty from the former is determined from the upper and lower limits [37] of the most uncertain parameter in the model. This leads to an uncertainty of $28 \mathrm{MeV}$ due to parton luminosity and $12 \mathrm{MeV}$ due to uncertainty in the transverse momentum of the $W$ boson in the model. There are several PDF models currently in use. The uncertainty due to variation in PDF's is determined by using different PDF's, including MRSA [40], CTEQ4M and CTEQ5M [41], and finding the largest excursion from the value of $\Gamma_{W}$ deter- mined using the MRST PDF set [42], leading to a variation of $27 \mathrm{MeV}$. The value quoted for $\Gamma_{W}$ is determined using the MRST PDF's. We chose MRST so that the results can be consistent with $\mathrm{D} \varnothing$ mass analysis [15].

\section{B. Backgrounds}

Backgrounds to $W \rightarrow e \nu$ can affect the shape of the $m_{T}$ spectrum and skew the measurement of $\Gamma_{W}$. We account for this by estimating the background as a function of $m_{T}$ and adding this to the $m_{T}$ distribution of the $W$ boson from the Monte Carlo. The three dominant background sources are multijet events, $Z \rightarrow e e$, and $W \rightarrow \tau \nu$ decay products. The following describes how the backgrounds are estimated [28].

A large potential source of background is due to multijet events in which one jet is misidentified as an electron and the energy in the event is mis-measured, thereby yielding large $\mathbb{E}_{T}$. This background is estimated using jet events from data, following the procedure called the "matrix method," described in Refs. [25,28,32]. The method uses two sets of data, each containing both signal and background. The first data set corresponds to the $W$ data sample in this analysis. The second set contains a different mix of signal and background which is obtained with loose electron criteria (described in Sec. III A). We summarize below the essence of this method used to estimate the multijet background.

The number of multijet background $\left(N_{\mathrm{BG}}^{W}\right)$ events in the tight electron $W$ data sample is given by

$$
N_{\mathrm{BG}}^{W}=\epsilon_{j} \frac{\epsilon_{s} N_{l}-N_{t}}{\epsilon_{s}-\epsilon_{j}},
$$

where $N_{l}$ and $N_{t}$ are the number of events in the $W$ boson samples satisfying loose and tight electron criteria, respectively. The tight electron efficiency $\epsilon_{s}$ is the fraction of loose electrons that pass tight electron criteria, as determined by the $Z$ boson sample, where one electron is required to pass the tight selection criteria and the other serves as an unbiased probe for determining relative efficiencies. The electron efficiency is obtained to be $\boldsymbol{\epsilon}_{s}=(86.3 \pm 1.2) \%$. The jet efficiency $\epsilon_{j}$ is the fraction of loose "electrons" found in multijet events that also pass tight electron criteria. This sample is required to have $\boldsymbol{E}_{T} \leqslant 15 \mathrm{GeV}$ to minimize the number of $W$ bosons contained in it. The result is $\epsilon_{j}=(5.83 \pm 0.25) \%$. Both $\epsilon_{s}$ and $\epsilon_{j}$ are found to be constant within statistical error as a function of $W$ transverse mass. Once $\epsilon_{s}$ and $\epsilon_{j}$ are determined, we can extract the background-event distribution. The "electron" and "neutrino" transverse momenta and energies are used to form the transverse mass, and this distribution is shown in Fig. 4. The total multijet background is estimated to be $368 \pm 32$ events in the region $m_{T}$ $<200 \mathrm{GeV}$, with $25.4 \pm 2.2$ events in the range $90 \mathrm{GeV}$ $<m_{T}<200 \mathrm{GeV}$.

The background sample is smoothed in the region $85 \mathrm{GeV}<m_{T}<200 \mathrm{GeV}$. We fit the distribution to an exponential function of the form $f_{\mathrm{BG}}=\exp \left(a_{0}+a_{1} x+a_{2} x^{2}+a_{3} x^{3}\right)$. The fitting parameters $a_{0}, a_{1}, a_{2}$, and $a_{3}$ [43] are used to generate the background distribution for the fit to the signal. For bins outside the fitted region, we use the original data itself, as shown in Fig. 4. 


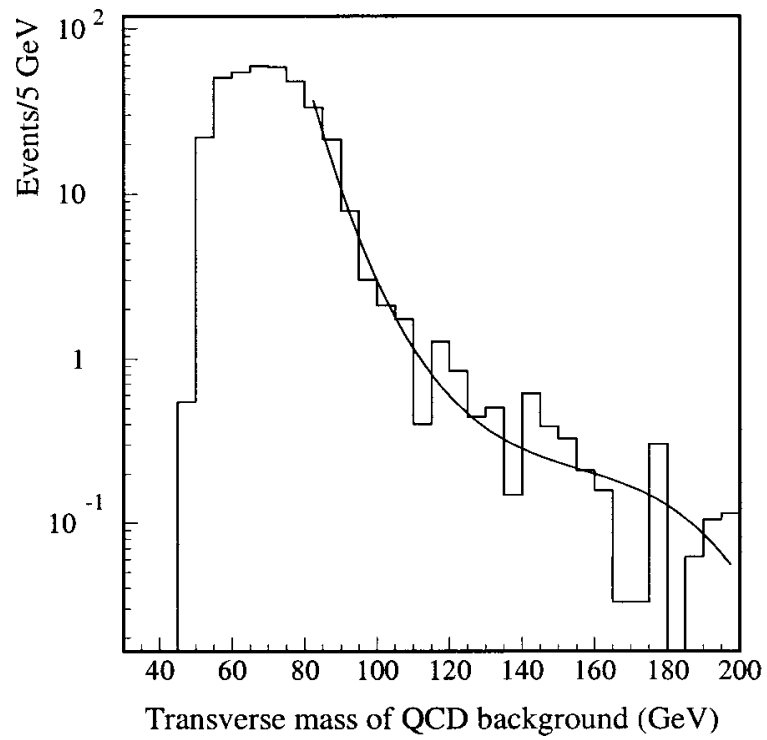

FIG. 4. The transverse mass distribution for the multijet background. The line represents the results of the fit described in the text.

Another source of background is due to $Z \rightarrow e e$ events in which one electron is undetected. This results in a momentum imbalance, with the event now being topologically indistinguishable from $W \rightarrow e \nu$ events. This background is also estimated using Monte Carlo events. The number of such $Z$ boson events present in the $W$ boson sample is calculated by applying the $W$ boson selection criteria to $\mathrm{MC} Z \rightarrow e e$ events generated using HERWIG [44] and processed through a GEANT [45] based simulation of the D $\varnothing$ detector, and then overlaid with events from random $p \bar{p}$ crossings. This is done to simulate the effect of the luminosity on the underlying event. Out of a total of $8870 Z \rightarrow e e$ events, 48 pass the $W$ boson event selection. Normalizing the Monte Carlo sample to the size of the data sample for equivalent luminosity, we estimate that there are $102 \mathrm{Z} \rightarrow e e$ events in the data sample.

$W \rightarrow \tau \nu$ events in which the $\tau$ decays into an electron and two neutrinos are indistinguishable from $W \rightarrow e \nu$ events on an event-by-event basis. Because $\tau$ undergoes a three-body decay, leading to a softer electron relative to $W \rightarrow e \nu$ events, the acceptance is reduced greatly by the standard $E_{T}$ selection criteria. The size of this background is small, and it tends to add events with low values of $m_{T}$. This background is determined using the $W \rightarrow e \nu$ Monte Carlo, modified to include the decay of the $\tau$ lepton. The events are then passed through the same detector simulation used to model the $W$ $\rightarrow e \nu$ signal.

The shape and total amount of background affect the fit used to determine the width of $W$ boson. To estimate the uncertainty in $\Gamma_{W}$ due to the uncertainty in absolute background, we scale up (and down) the fitted number of background events by an amount that corresponds to the total uncertainty in the background. This gives an uncertainty of $15 \mathrm{MeV}$ for $\Gamma_{W}$ extracted from the region $90 \mathrm{GeV}<m_{T}$ $<200 \mathrm{GeV}$. To estimate the uncertainty in $\Gamma_{W}$ from the uncertainty in the shape of the background spectrum, we per- form an ensemble study in which background is generated using a multinomial distribution. The multinomial distribution is defined by

$$
P\left(N_{1}, N_{2}, \ldots, N_{c h}\right)=N_{\text {total }} \prod_{i=1}^{c h} \frac{p_{i}^{N_{i}}}{N_{i} !}
$$

where $N_{\text {total }}$ is the total number of background events, $c h$ is the number of the bins, $p_{i}$ is the original distribution, and $N_{i}$ is numbers of events in $i$ th bin. The total background $N_{\text {total }}$ is kept at its central value, while the number of background events in each bin is allowed to fluctuate. The $W$ boson width is then recalculated with the new background distribution. The variation in $\Gamma_{W}$ is taken as the uncertainty. We found that this is $39 \mathrm{MeV}$ for the fitted region of $m_{T}$.

\section{Likelihood fitting}

We generate a set of Monte Carlo $m_{T}$ templates with $\Gamma_{W}$ varying from $1.55 \mathrm{GeV}$ to $2.75 \mathrm{GeV}$ at intervals of $50 \mathrm{MeV}$. These templates are normalized to the number of events in the region of $m_{T}<200 \mathrm{GeV}$. The background distributions of multijet and $Z \rightarrow e e$ events are added to the templates and a binned likelihood is calculated for data. The $m_{T}$ bin size is 5 $\mathrm{GeV}$. The fitting region is chosen to be $90 \mathrm{GeV}<m_{T}$ $<200 \mathrm{GeV}$ to minimize the systematic uncertainty. From the dependence of the likelihood on $\Gamma_{W}$, we obtain the $W$ boson width and its error as $\Gamma_{W}=2.23_{-0.14}^{ \pm 0.15}$ (stat) GeV. The combined uncertainty, taking the statistical and systematic uncertainties contribution in quadrature, yields the result $\Gamma_{W}$ $=2.23_{-0.14}^{+0.15}$ (stat) \pm 0.10 ( syst) $\mathrm{GeV}=2.23_{-0.17}^{+0.18} \mathrm{GeV}$. The $\chi^{2}$ for the best fit is an acceptable 25.9 for 22 degrees of freedom, corresponding to a probability of $26 \%$. A comparison of the observed spectrum to the probability density function in the fitting region through a Kolmogorov-Smirnov test, which compares the observed cumulative distribution function for a variable with a specified theoretical distribution, yields $\kappa=0.434$, which is evidence of a good fit.

Figure 5 shows a fit to the likelihood, which corresponds to a fourth-order polynomial fit that determines the peak position. Figure 6 shows the $m_{T}$ spectrum for the data, the normalized MC sample, and the background.

As a consistency check of the fitting method, we also determine the $W$ boson width from the ratio of the number of events in the fitting region of $90 \mathrm{GeV} \leqslant m_{T} \leqslant 200 \mathrm{GeV}$ to the number of events in the entire spectrum. This yields $\Gamma_{W}=2.22 \pm 0.14$ (stat) $\mathrm{GeV}, \quad$ compared to $\Gamma_{W}$ $=2.23_{-0.14}^{+0.15}$ (stat) $\mathrm{GeV}$ for the independent maximum likelihood fit in the same region. All results show good agreement.

Sources of systematic uncertainties in the determination of the $W$ boson width are those that can affect the shape of the transverse mass distribution. These include the uncertainties from input parameters to the MC program and from background estimation. Details can be found in corresponding section of the parameters and in Ref. [28]. Table I lists all the important sources of systematic uncertainty for the decay width of the $W$ boson. 


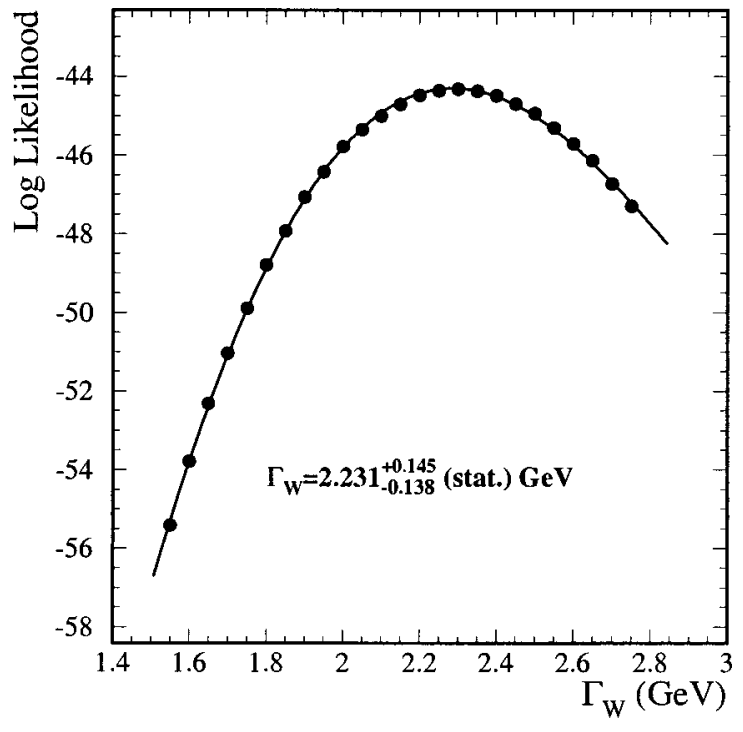

FIG. 5. Results of the log-likelihood fit of the data to Monte Carlo templates for different $\Gamma_{W}$.

Comparing to the SM prediction of $\Gamma(W)=2.0921$ $\pm 0.0025 \mathrm{GeV}$, we find the difference between SM prediction and our measurement to be $0.24_{-0.17}^{+0.18} \mathrm{GeV}$, which is the width for the $W$ boson to decay into final states other than the two lightest quark doublets and the three lepton doublets. We set a 95\% confidence level upper limit on the $W$ boson width to non-SM final states. Assuming the uncertainty is Gaussian, we set a 95\% confidence level upper limit on the invisible partial width of the $W$ boson to be $0.59 \mathrm{GeV}$. Under the assumption that there is no correlation between indirect measurement and direct measurement of the $W$ boson decay

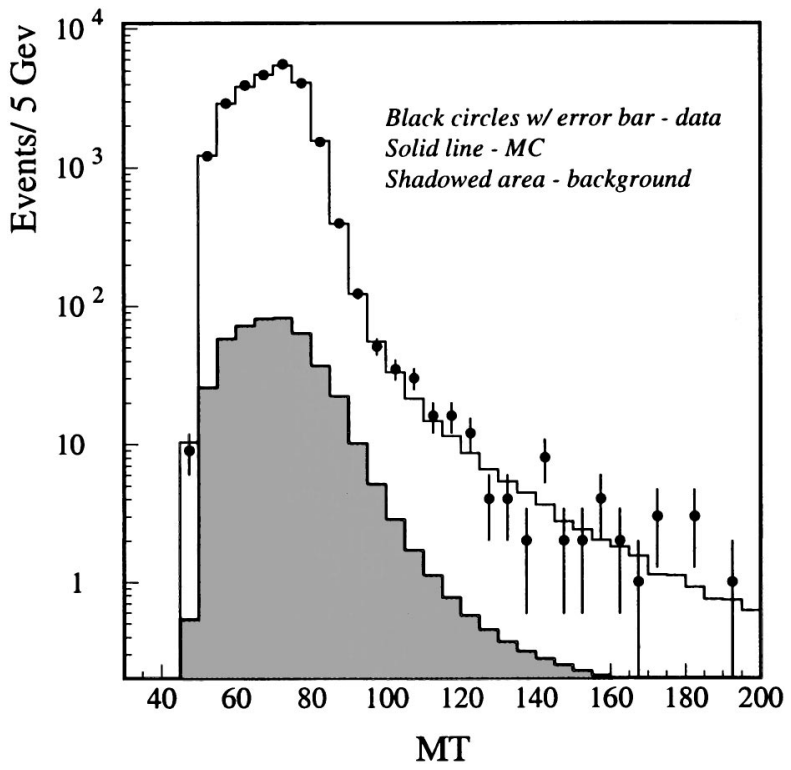

FIG. 6. Comparison of data to the Monte Carlo templates for the best fit. The black circles with error bars are the data. The solid line of the histogram corresponds to the MC templates with $\Gamma(W)$ $=2.23 \mathrm{GeV}$ normalized to the expected number of $W$ boson events. The shadowed area is the background.
TABLE I. Systematic uncertainties and the total uncertainty on the $W$ boson width measurement.

\begin{tabular}{lc}
\hline \hline Source & $\delta \Gamma_{W}(\mathrm{MeV})$ \\
\hline Hadronic energy resolution & 55 \\
EM energy scale & 41 \\
Background ensemble studies & 39 \\
Luminosity slope dependence & 28 \\
EM energy resolution & 27 \\
PDF & 27 \\
Hadronic energy scale & 22 \\
Background normalization & 15 \\
$W$ boson mass & 15 \\
Production model & 12 \\
Radiative correction & 10 \\
Selection bias & 10 \\
Angular calibration of $e$ trajectory & 9 \\
Total systematic uncertainty & 99 \\
Total statistical uncertainty & +145 \\
& -138 \\
Total uncertainty & +176 \\
& -170 \\
\hline \hline
\end{tabular}

width and within the framework of SM, we can combine both analyses and obtain $\Gamma_{W}=2.162 \pm 0.062 \mathrm{GeV}$. The $95 \%$ confidence level upper limit on the invisible partial width of the $W$ boson is $0.191 \mathrm{GeV}$.

\section{CONCLUSIONS}

We have directly measured the decay width of the $W$ boson by fitting the transverse mass in $W \rightarrow e \nu$ events in $p \bar{p}$ collisions at $1.8 \mathrm{TeV}$, and obtain

$$
\begin{aligned}
\Gamma_{W} & =2.23_{-0.14}^{+0.15}(\text { stat }) \pm 0.10(\text { syst }) \mathrm{GeV} \\
& =2.23_{-0.17}^{+0.18} \mathrm{GeV} .
\end{aligned}
$$

This result is consistent with the prediction of the standard model.

\section{ACKNOWLEDGMENTS}

We thank the staffs at Fermilab and collaborating institutions, and acknowledge support from the Department of Energy and National Science Foundation (USA), Commissariat à L'Energie Atomique and CNRS/Institut National de Physique Nucléaire et de Physique des Particules (France), Ministry for Science and Technology and Ministry for Atomic Energy (Russia), CAPES and CNPq (Brazil), Departments of Atomic Energy and Science and Education (India), Colciencias (Colombia), CONACyT (Mexico), Ministry of Education and KOSEF (Korea), CONICET and UBACyT (Argentina), The Foundation for Fundamental Research on Matter (The Netherlands), PPARC (United Kingdom), Ministry of Education (Czech Republic), A.P. Sloan Foundation, NATO, and the Research Corporation. 
[1] S. Glashow, Nucl. Phys. 22, 579 (1961).

[2] S. Weinberg, Phys. Rev. Lett. 19, 1264 (1967).

[3] A. Salam, in Elementary Particle Theory, edited by N. Svartholm (Almqvist and Wiksells, Stockholm, 1969), p. 367.

[4] W. Bardeen, H. Fritzsch, and M. Gell-Mann, in Scale and Conformal Symmetry in Hadron Physics, edited by R. Gatto (Wiley, New York, 1973), p. 139.

[5] D. Gross and F. Wilczek, Phys. Rev. D 8, 3633 (1973).

[6] S. Weinberg, Phys. Rev. Lett. 31, 494 (1973).

[7] UA1 Collaboration, G. Arnison et al., Phys. Lett. 122B, 103 (1983).

[8] UA2 Collaboration, P. Bagnaia et al., Phys. Lett. 122B, 476 (1983).

[9] UA1 Collaboration, G. Arnison et al., Phys. Lett. 126B, 398 (1983).

[10] UA2 Collaboration, P. Bagnaia et al., Phys. Lett. 129B, 130 (1983).

[11] Particle Data Group, D. E. Groom et al., Eur. Phys. J. C 15, $1-878$ (2000).

[12] J. Rosner, M. Worah, and T. Takeuchi, Phys. Rev. D 49, 1363 (1994).

[13] Particle Data Group, R. M. Barnett et al., Phys. Rev. D 54, 1 (1996)

[14] CDF Collaboration, F. Abe et al., Phys. Rev. D 43, 2070 (1991).

[15] DØ Collaboration, B. Abbott et al., Phys. Rev. D 58, 092003 (1998).

[16] L3 Collaboration, M. Acciarri et al., Phys. Lett. B 413, 176 (1997).

[17] ALEPH Collaboration, R. Barate et al., Phys. Lett. B 422, 384 (1998).

[18] OPAL Collaboration, K. Ackerstaff et al., Eur. Phys. J. C 1, 395 (1998).

[19] Delphi Collaboration, P. Abreu et al., Eur. Phys. J. C 2, 581 (1998).

[20] A. D. Martin, R. G. Roberts, and W. J. Stirling, Phys. Lett. B 306, 147 (1993); 309, 492 (1993).

[21] LEP Electroweak Working Group, CERN Report No. CERNEP-2001-098, hep-ex/0112021.

[22] UA1 Collaboration, C. Albajar et al., Phys. Lett. B 253, 503 (1991).

[23] UA2 Collaboration, J. Alitti et al., Phys. Lett. B 276, 365 (1992).

[24] CDF Collaboration, F. Abe et al., Phys. Rev. D 52, 2624 (1995).

[25] DØ Collaboration, B. Abbott et al., Phys. Rev. D 61, 072001 (2000).
[26] V. D. Barger and R. J. N. Phillips, in Collider Physics, Vol. 71 of Frontiers in Physics (Addison-Wesley, New York, 1987).

[27] CDF Collaboration, F. Abe et al., Phys. Rev. Lett. 85, 3347 (2000). CDF measured the $W$ boson width in both the $e \nu$ and $\mu \nu$ channels. The number reported is their combined result.

[28] Qichun Xu, Ph.D. thesis, University of Michigan, 2001; http:// www-d0.fnal.gov/results/publications_talks/thesis/xu/ qichun_thesis.html

[29] The origin of the coordinate system is the reconstructed position of $p \bar{p}$ interaction when describing the interaction, and the geometrical center of the detector when describing the detector. It refers to the detector here.

[30] DØ Collaboration, S. Abachi et al., Nucl. Instrum. Methods Phys. Res. A 338, 185 (1994).

[31] DØ Collaboration, B. Abbott et al., Phys. Rev. D 58, 052001 (1998).

[32] DØ Collaboration, V. M. Abazov et al., Phys. Lett. B 513, 292 (2001).

[33] DØ Collaboration, S. Abachi et al., Phys. Rev. D 52, 4877 (1995).

[34] Eric M. Flattum, Ph.D. thesis, Michigan State University, 1996, http://www-d0.fnal.gov/results/publications_talks/ thesis/flattum/eric_thesis.html

[35] Ian Malcolm Adam, Ph.D. thesis, Columbia University, 1997, http://www-d0.fnal.gov/results/publications_talks/thesis/adam/ ian_thesis_all.html

[36] C. Balazs and C. P. Yuan, Phys. Rev. D 56, 5558 (1997).

[37] G. A. Ladinsky and C. P. Yuan, Phys. Rev. D 50, 4239 (1994).

[38] P. B. Arnold and M. H. Reno, Nucl. Phys. B319, 37 (1989); B330, 284E (1990); R. J. Gonsalves, J. Pawlowski, and C.-F. Wai, Phys. Rev. D 40, 2245 (1989).

[39] DØ Collaboration, S. Abachi et al., Phys. Rev. Lett. 77, 3309 (1996); D $\varnothing$ Collaboration, B. Abbott et al., Phys. Rev. D 58, 012002 (1998).

[40] A. D. Martin, W. J. Stirling, and R. G. Roberts, Phys. Lett. B 354, 155 (1995).

[41] The Coordinated Theoretical-Experimental Project on QCD, http://www.phys.psu.edu/cteq

[42] A. D. Martin, R. G. Roberts, W. J. Stirling, and R. R. Thorne, hep-ph/0110215.

[43] We found the fitting parameters as $a_{0}=(3.9153 \pm 0.0012)$ $\times 10^{1}, \quad a_{1}=(-7.5100 \pm 0.0044) \times 10^{-1}, \quad a_{2}=(4.7087$ $\pm 0.0041) \times 10^{-3}$, and $a_{3}=(-1.00461 \pm 0.00095) \times 10^{-5}$.

[44] G. Marchesini et al., Comput. Phys. Commun. 67, 465 (1992).

[45] F. Carminati et al., GEANT Users Guide, CERN Program Library W5013, 1991. 